\title{
Psychometrische Verfahren in der Psychotherapie
}

\author{
Rolf-Dieter Stieglitz \\ Abteilung für Klinische Psychologie und Psychiatrie, Fakultät für Psychologie, Universität Basel, Schweiz
}

\section{Schlüsselwörter \\ Therapiebegleitende Diagnostik · Psychometrische Verfahren - Psychotherapie · Ratingskalen}

\begin{abstract}
Zusammenfassung
Psychotherapie als zielgerichteter Prozess beinhaltet die Notwendigkeit der Überprüfung der Zielerreichung, wozu in den letzten Jahrzehnten vielfältige Entwicklungen erkennbar sind. Die Entwicklung psychometrischer Verfahren allgemein wie speziell für einzelne Störungsgruppen ist hier zu nennen. Instrumente können in verschiedenen Phasen der Therapie in Abhängigkeit von den jeweiligen Assessmentzielen zur Anwendung kommen. Sie dienen vor allem der Kontrolle des Therapieverlaufs und des Therapieerfolgs. Der Beitrag gibt einen Überblick zu Instrumenten und ihren Anwendungsmöglichkeiten und weist auf bestehende Defizite hin.
\end{abstract}

\section{Einleitung}

Den verschiedenen Definitionen der Psychotherapie gemeinsam ist die Betonung der Zielgerichtetheit, was impliziert, dies auch zu belegen und zu objektivieren. Hierzu können im Rahmen einer therapiebegleitenden Diagnostik psychometrische Verfahren zum Einsatz kommen. In Abgrenzung zu sogenannten Adhoc-Instrumenten, die oft für eine spezielle Fragestellung zusammengestellt werden, handelt es sich bei psychometrischen Instrumenten um Instrumente, die nach spezifischen Kriterien entwickelt und evaluiert wurden.

Historisch gesehen spielte die therapiebegleitende Diagnostik zunächst eine eher untergeordnete bzw. keine Rolle. In tiefenpsychologischen bzw. psychodynamischen Ansätzen tauchte sie anfänglich gar nicht auf, in systemischen Ansätzen ebenfalls nicht. Im Rahmen der Verhaltenstherapie spielte sie bei verhaltensanalytischen Überlegungen zunächst eher eine randständige Rolle [vgl. z.B. Schulte, 1974]. Erst durch die Arbeitsgruppe um Beck [z.B. Beck et al., 2006] gewannen psychometrische Verfahren an Bedeutung. Seit Mitte der 1960er Jahre wurde eine Vielzahl von

\author{
Keywords \\ Therapy-related assessment · Psychometric methods . \\ Psychotherapy · Rating scales
}

\section{Summary}

Psychometric Methods in Psychotherapy

Psychotherapy is a goal-oriented process. As such, it is essential to assess the achievement of therapeutic targets. Over the last decades, various developments have occurred in this area. Particularly important are the developments of psychometric methods, both in general and disorder-specific measures. Assessment tools can be applied in different phases of therapy, depending on the particular assessment purposes. They serve mainly as control for therapy process and evaluation. This article gives an overview of tools and their potential applications, while simultaneously highlighting remaining deficits.

Instrumenten im Kontext der kognitiven Therapie der Depression nach Beck entwickelt [vgl. z.B. Stieglitz, 2008]. In der Gesprächspsychotherapie spielten psychometrische Verfahren seit jeher eine Rolle im Kontext der Prozess- und Ergebnisforschung [vgl. Höger, 2006a,b]. Seit ca. 30-40 Jahren kann man jedoch generell eine zunehmende Bedeutung von diagnostischen Verfahren in der Psychotherapie erkennen.

In der Psychopharmakotherapie waren derartige Bemühungen bereits mit der Entdeckung unter anderem von Antidepressiva und Neuroleptika in den 1950er Jahren zu beobachten, die erstmalig deutlich verbesserte Behandlungsoptionen psychischer Störungen zur Folge hatten. Das Fehlen geeigneter Instrumente zum Nachweis der Wirksamkeit löste in den 1960er Jahren verschiedene Instrumentenentwicklungen aus. Exemplarisch genannt sei die Entwicklung des AMDP-Systems (Arbeitsgemeinschaft für Methodik und Dokumentation in der Psychiatrie) zur Erfassung psychischer und somatischer Symptome psychiatrischer Störungen [Baumann und Stieglitz, 1983]. Ein Beleg dieser Entwicklung war auch die Herausgabe des sogenannten CIPSManuals [Collegium Internationale Psychiatriae Scalarum; CIPS,

\section{KARGER \\ Fax +497614520714 \\ Information@Karger.com}

www.karger.com (c) 2014 S. Karger GmbH, Freiburg

$1016-6262 / 14 / 0241-0056 \$ 39.50 / 0$

Accessible online at:

www.karger.com/ver
Prof. Dr. rer. nat. Rolf-Dieter Stieglitz, Dipl.-Psych.

Abteilung für Klinische Psychologie und Psychiatrie

Fakultät für Psychologie, Universität Basel

Missionsstrasse 62a, 4055 Basel, Schweiz

rolf-dieter.stieglitz@upkbs.ch 
1977] mit der erstmaligen Zusammenstellung der wichtigsten Instrumente für die Forschung. Die Zunahme von Instrumenten machte es notwendig, diejenigen auszuwählen, die sich für bestimmte Störungsgruppen als besonders geeignet erwiesen hatten. Hierzu wurden zu verschiedenen Substanzklassen wie Störungsgruppen seit Ende der 1980er Jahre Konsensuskonferenzen abgehalten [z.B. Angst et al., 1989].

Parallele Entwicklungen gab es auch aufseiten der Psychotherapie. Exemplarisch genannt sei hier die Monografie von Waskow und Parloff [1975], die damit das Ziel verfolgten, eine verbindliche Testbatterie zur Evaluation psychotherapeutischer Behandlungen einzuführen. Der Band besteht aus insgesamt 21 Kapiteln, von denen sich 19 mit psychotherapeutisch relevanten Outcome-Maßen beschäftigen. Daraus abgeleitet, wurde eine sogenannte Core Battery empfohlen. An der damaligen Diskussion beteiligten sich bis heute bekannte Vertreter der Psychotherapie und Psychiatrie wie Kanfer, Kiesler, Endicott, Strupp, Lorr, Fiske, Spitzer oder Luborsky. Leider konnten sich die Vorschläge nicht durchsetzen. Ähnliches versuchten später Strupp et al. [1997]. Sie schlugen sogar verschiedene Core Batteries für die Störungsbereiche Angst (Posttraumatische Belastungsstörung (PTBS), Soziale Phobie), Affektive Störungen und Persönlichkeitsstörungen vor. Auch dieser Vorschlag konnte sich nicht etablieren, weder in Forschung noch in Praxis. In der Folgezeit wurden nur vereinzelt systematische Vorschläge von Verfahren für einzelne Störungsbereiche gemacht (z.B. von Bandelow und Margraf [1994] für den Angstbereich). Von Laireiter [2000] wurde für den deutschsprachigen Bereich erstmals der interessante Versuch unternommen, eine umfassende Bestandsaufnahme der Diagnostik in der Psychotherapie zu liefern, wobei der Schwerpunkt des Bandes in der Darstellung der Diagnostik der einzelnen psychotherapeutischen Schulen lag [vgl. hierzu auch Linster et al., 2003]. Hierbei zeigen sich große Unterschiede hinsichtlich des Stellenwerts der Diagnostik. Speziell die Verhaltenstherapie misst ihr den größten Stellwert bei, unter anderem bedingt durch ihre empirische Orientierung. Jedoch auch in tiefenpsychologischen und psychodynamischen Ansätzen gewinnt die Diagnostik gerade auch in den letzten Jahren an Bedeutung [vgl. z.B. Doering und Hörz, 2012], wenngleich psychometrische Verfahren im eigentlichen Sinne im Vergleich zu Interviewverfahren weniger Anwendung finden.

Seit einigen Jahren ist insgesamt eine zunehmend stärker werdende Verbindung zwischen Diagnostik und Therapie erkennbar. In der mittlerweile etablierten Reihe des Hogrefe-Verlags «Fortschritte der Psychotherapie» findet sich z.B. in den Bänden zu Störungen eine Rubrik «Diagnostik und Indikation», wobei sich je nach Band unterschiedlich differenzierte Ausführungen darüber finden, welche Instrumente zum Einsatz kommen können. Im «Handbook of Assessment and Treatment Planning» von Antony und Barlow [2011] zeigt sich bereits im Titel die enge Verzahnung von Diagnostik und Therapie. In 13 störungsspezifischen Kapiteln werden differenziert unterschiedliche Verfahrensgruppen vorgestellt und praktische Empfehlungen für die Anwendung gegeben. Speziell die Abschnitte «From Assessment to Treatment» unterstreichen dies. In deutschsprachigen Lehrbüchern zur Psychotherapie wird die Thematik der Diagnostik in der Psychotherapie jedoch oft immer noch stiefmütterlich abgehandelt. Exemplarisch als Ausnahmen genannt seien die Lehrbücher von Rief et al. [2006], Lutz [2010] und Margraf und
Schneider [2009]. Bei Rief et al. [2006] findet sich ein umfassender Abschnitt zur Diagnostik mit speziellen Kriterien zur Auswahl von Verfahren. Bei Lutz [2010] wird die indikationsorientierte Diagnostik von der therapiebegleitenden bzw. Prozessdiagnostik abgegrenzt. Bei Margraf und Schneider [2009] werden die vielfältigen Facetten der Diagnostik in der Psychotherapie dargestellt. Zudem wird in einem Materialienband eine große Anzahl von Instrumenten zur störungsübergreifenden wie störungsspezifischen Diagnostik zusammengestellt [Meinlschmidt et al., 2012].

Konkrete Vorschläge für die praktische Anwendung psychometrischer Verfahren finden sich erstmals umfassend bei Grawe und Braun [1994]. In einem Rahmenmodell psychotherapeutischer Diagnostik, dem sogenannten Figurationsanalytischen Ansatz, schlagen die Autoren verschiedene Möglichkeiten der Kontrolle von Prozess- und Ergebnisqualität vor. Darin wird versucht, die Veränderung eines einzelnen Patienten in Bezug auf die relevante Bezugs- oder Referenzgruppe abzubilden, d.h. eine Gruppe vergleichbarer Psychotherapiepatienten (Figuration). Unterschieden wird dabei zwischen Effekt-, Zustands- und Prozessfiguration unter Verwendung psychometrischer Verfahren. Effektkonfiguration umfasst die Bewertung der Veränderung im Prä-Post-Vergleich, Zustandsfiguration den Ausgangszustand des Patienten bei Therapiebeginn und Prozessfiguration die Prozessqualität der Therapie, erfasst mittels sogenannter Stundenbögen [vgl. hierzu auch Stieglitz und Hiller, 2014]. Diese beinhalten unter anderem die Erfassung der Zufriedenheit mit der Therapie sowie Fortschritte innerhalb und außerhalb der Therapie. Die computerbasierte Auswertung ermöglicht eine schnelle und zeitnahe Rückmeldung an den Therapeuten im Hinblick auf mögliche Veränderungen innerhalb der Therapie. Dieses interessante, aber eher aufwändige Procedere lässt sich in der Praxis durch selbstkonzipierte therapiebegleitende Diagnostik unter Auswahl geeigneter Instrumente vereinfachen.

Unter einer therapiebegleitenden Diagnostik (zum Teil auch als therapiebezogen bezeichnet) versteht man alle diagnostischen Maßnahmen, die zu Beginn, im Verlauf und am Ende einer Therapie durchgeführt werden, mit dem Ziel der Beantwortung von Fragen, die für die jeweilige (Therapie-)Phase spezifisch sind (Tab. 1). Während zu Beginn die Statusbestimmung als Ausgangspunkt für eine spätere Veränderungsbewertung erfolgt, geht es im Verlauf um die kontinuierliche Evaluation des Therapieprozesses und am Ende um die Gesamtbeurteilung des Therapieerfolgs. Ziel aller Erhebungen ist es, Informationen für die Optimierung der Behandlung zu bekommen [vgl. auch Stieglitz und Hiller, 2014]. Bei der Katamnese gilt es festzustellen, inwieweit der Therapieerfolg gegenüber dem Ende der Behandlung stabil geblieben ist. Laireiter [2000] wählt eine etwas andere Nomenklatur und spricht von einer Indikationsorientierten Diagnostik (vor und zu Beginn der Therapie), Verlaufsund Prozessdiagnostik (im Verlauf der Therapie) und einer Evaluativen Diagnostik (am Ende und nach der Therapie).

Die Anwendung psychometrischer Verfahren in der Psychotherapie zeigt allmählich eine steigende Tendenz, was auf verschiedene Faktoren zurückzuführen ist wie die Zunahme verfügbarer Verfahren, die auch therapierelevant sind [vgl. z.B. Brähler et al., 2003; Voderholzer et al., 2014], die zunehmende Forderung, die Qualität der Behandlung zu dokumentieren (Qualitätssicherung/-management) [vgl. z.B. Linster et al., 2003] und das Erkennen des Nutzens für die konkrete Therapie. 
Tab. 1. Therapiebegleitende Diagnostik: Phasen und Assessment-Ziele

\begin{tabular}{ll}
\hline Phase & Assessment-Ziele, Beispiele \\
\hline Erstgespräch & Indikation, Motivation \\
Prä-Messung & Status zu Beginn der Therapie, Informationen für die Behandlung (Therapieplanung, Problemanalyse) \\
Prozess-Messung & Beurteilung Therapieverlauf, Informationen für und über die Behandlung \\
Post-Messung & Beurteilung des Therapieerfolgs/-effekts \\
Katamnese & Beurteilung der Stabilität und des Therapieerfolgs, Generalisierung der Erfolge \\
\hline
\end{tabular}

Abb. 1. Diagnostische Ebenen im Kontext der

\begin{tabular}{|c|c|c|c|c|c|}
\hline Ebene 1 & $\begin{array}{r}\text { kategoriale Diagn } \\
\text { nach ICD-10 oder D } \\
\text { (z.B. mittels struktu } \\
\text { Interviews) }\end{array}$ & & & & \\
\hline Ebene 2 & $\begin{array}{l}\text { Globalbewertung } \\
\text { (z.B. mittels CGI } \\
\text { oder GAF) }\end{array}$ & & \multicolumn{2}{|c|}{$\begin{array}{l}\text { allgemeine und } \\
\text { spezifische } \\
\text { Psychopathologie } \\
\text { (z.B. mittels SCL-90-R) }\end{array}$} & $\begin{array}{l}\text { allgemeine Aspekte } \\
\text { der Persönlichkeit } \\
\text { (z.B. mittels PSSI) }\end{array}$ \\
\hline \multirow[t]{2}{*}{ Ebene 3} & $\begin{array}{c}\text { Beeinträchtigung } \\
\text { (z.B. mittels } \\
\text { Sheehan-Skala) }\end{array}$ & & \multicolumn{2}{|c|}{$\begin{array}{c}\text { Lebensqualität } \\
\text { (z.B. mittels SF-36) }\end{array}$} & \\
\hline & \multicolumn{5}{|c|}{ therapiebezogene, spezifische Diagnostik } \\
\hline Ebene 4 & psychodynamisch & & $\begin{array}{l}\text { Itens- } \\
\text { eutisch }\end{array}$ & $\begin{array}{r}\text { gespräch } \\
\text { therap }\end{array}$ & systemisch \\
\hline
\end{tabular}
Psychotherapie.

Persönlichkeit von Bedeutung, wozu sich z.B. das PersönlichkeitsStil- und Störungs-Inventar [PSSI; Kuhl und Kazén, 2006] anbietet, welches sich eng an der Definition der DSM-IV-Subtypen der Persönlichkeitsstörungen orientiert. Auf Ebene 3 kommen Verfahren zum Einsatz, die über die Psychopathologie und assoziierte Aspekte hinaus klinisch relevante Bereiche im Sinne von «Folgen» der Störung erfassen, wie Beeinträchtigungen oder Lebensqualität. Ersterer Bereich lässt sich z.B. einfach mit der häufig eingesetzten Sheehan Disability Scale [SDS; Hoyer und Margraf, 2003] erfassen, während für den Bereich der Lebensqualität viele verschiedene Instrumente zur Verfügung stehen. Häufig eingesetzt wird der Fragebogen zum Gesundheitszustand [SF-36; Morfeld et al., 2011]. Auf Ebene 4 kommen dann die verschiedenen therapiebezogenen Verfahren zur Anwendung, wobei hier eine Differenzierung hinsichtlich der Therapieschulen notwendig ist [vgl. Laireiter, 2000; Linster et al., 2003].

Bezogen auf die im eigentlichen Sinne therapiebegleitende Diagnostik lassen sich weitere Unterscheidungen treffen. Hinsichtlich der allgemeinen Psychopathologie (vgl. auch Ebene 2 in Abbildung 1) geht es um die differenzierte Erfassung klinisch relevanter Bereiche über die individuelle und spezifische Problematik des Patienten hinaus. Störungsspezifische Verfahren dienen der Erfassung der im Zentrum der aktuellen Behandlung stehenden Symptomatik, während Verfahren einer therapiebezogenen Diagnostik ergänzend dazu spezifische, für die jeweils gewählte Therapie relevante Informationen liefern (z.B. Erfassung von Vermeidungsverhalten oder Kognitionen im Rahmen einer kognitiv- 
Tab. 2. Skalen zur störungsübergreifenden Diagnostik (Auswahl)

\begin{tabular}{|c|c|c|c|c|}
\hline Bereich & Verfahren & $\mathrm{S} / \mathrm{F}$ & Kennzeichen & Kommentar \\
\hline \multirow[t]{3}{*}{$\begin{array}{l}\text { Allgemeine } \\
\text { Psychopathologie }\end{array}$} & $\begin{array}{l}\text { Symptom-Checklist-90 } \\
\text { [SCL-90 }{ }^{\circledR} \text {; Franke, 2013] }\end{array}$ & $\mathrm{S}$ & $\begin{array}{l}90 \text { Items, } 9 \text { Subskalen, } \\
3 \text { globale Skalen }\end{array}$ & $\begin{array}{l}\text { Mehrdimensionalität fraglich, } \\
\text { Kurzformen vorhanden }\end{array}$ \\
\hline & $\begin{array}{l}\text { Outcome Questionnaire } \\
\text { [OQ-45.2; Lambert et al., 2002] }\end{array}$ & $\mathrm{S}$ & $\begin{array}{l}45 \text { Items, } 3 \text { Subskalen, } \\
\text { Globalwert }\end{array}$ & $\begin{array}{l}\text { im Kontext der Psychotherapie } \\
\text { entwickelt }\end{array}$ \\
\hline & $\begin{array}{l}\text { AMDP-System } \\
\text { [AMDP; AMDP, 2007] }\end{array}$ & $\mathrm{F}$ & $\begin{array}{l}140 \text { Symptome, } \\
9 \text { Subskalen }\end{array}$ & $\begin{array}{l}\text { eher für psychiatrische Patienten } \\
\text { geeignet; Interviewleitfaden } \\
\text { vorhanden [Fähndrich und Stieglitz, } \\
\text { 2007] }\end{array}$ \\
\hline \multirow[t]{2}{*}{ Beschwerden } & $\begin{array}{l}\text { Beschwerden-Liste - Revidierte Fassung } \\
\text { [B-LR; von Zerssen und Petermann, 2011a] }\end{array}$ & $\mathrm{S}$ & 20 Items, Gesamtwert & Parallelformen vorhanden \\
\hline & $\begin{array}{l}\text { Freiburger Beschwerdenliste } \\
\text { [FBL; Fahrenberg, 1994] }\end{array}$ & $\mathrm{S}$ & 80 Items, 10 Subkalen & Form FBL-R mit 9 Subskalen \\
\hline \multirow[t]{2}{*}{ Befindlichkeiten } & $\begin{array}{l}\text { Befindlichkeits-Skala - Revidierte Fassung } \\
\text { [Bf-SR; von Zerssen und Petermann, 2011b] }\end{array}$ & $\mathrm{S}$ & 24 Items, Gesamtwert & Parallelformen vorhanden \\
\hline & $\begin{array}{l}\text { Mehrdimensionaler Befindlichkeitsfragebogen } \\
\text { [MBFB; Steyer et al., 1997] }\end{array}$ & $\mathrm{S}$ & 24 Items, 3 Subskalen & bipolare Dimensionen \\
\hline
\end{tabular}

behavioralen Therapie). Die Erfassung von Therapieprozessen (z.B. mittels Stundenbögen; vgl. Stieglitz und Hiller [2014]) dient der Kontrolle des Verlaufs der Therapie im Hinblick z.B. auf die Patient-Therapeut-Beziehung. Therapierelevante Faktoren wie z.B. die Psychotherapiemotivation lassen sich gleichfalls mittels psychometrischer Verfahren erfassen (indikationsorientierte Diagnostik; vgl. Laireiter [2000]). Gleiches gilt auch für den Therapierfolg, wenngleich hier ergänzend oft therapiezielorientierte Ansätze zum Tragen kommen (z.B. Goal Attainment Scaling (GAS); vgl. Watzke et al. [2014]). Die nachfolgenden Ausführungen fokussieren auf die Bewertung der Symptomatologie im Querschnitt wie im Therapieverlauf, wobei zwischen der allgemeinen Symptomatologie und der störungsspezifischen sowie einer mit der speziellen Therapie assoziierten Symptomatik/Problematik unterschieden werden soll. Beim Einsatz von psychometrischen Verfahren ist zu beachten, in welchem Zeitraster eine Erfassung sinnvoll ist (Abb. 2). Je nach unterschiedlichem Bezugspunkt lassen sich Aussagen treffen, z.B. zum Therapiefortschritt oder Therapieergebnis. Die Wahl der Messzeitpunkte ist von verschiedenen Faktoren abhängig (unter anderem Konstrukt und Assessment-Ziel). So entscheidet z.B. das Konstrukt, wann ein sinnvoller Messabstand zu wählen ist. Bei der Erfassung z.B. der Depressivität wird dieser geringer sein als beispielsweise bei der Erfassung der Negativsymptomatik einer Schizophrenie. Ebenso ist das Assessment-Ziel ein wichtiger Faktor. Zur Dokumentation des Therapiefortschritts sind dichtere Messabstände zu wählen als zur Dokumentation des Therapieerfolgs.

Im Idealfall sollte zwischen Diagnostik und Therapie eine enge Verzahnung in Form eines Regelprozesses stattfinden [AssessTreat-Reassess-Adjust Treatment; Hunsley und Mash, 2005]. Der Ausgangspunkt der Behandlung dient der Quantifizierung des Status zu Beginn der Therapie (assess). Die sich anschließende Behandlung (treat) sollte nach einer gewissen Zeit evaluiert (reassess) und in Abhängigkeit vom Ergebnis gegebenenfalls modifiziert werden (adjust). Dies sollte immer dann erfolgen, wenn der Behandlungserfolg ungenügend ist (Stagnation oder Verschlechterung).

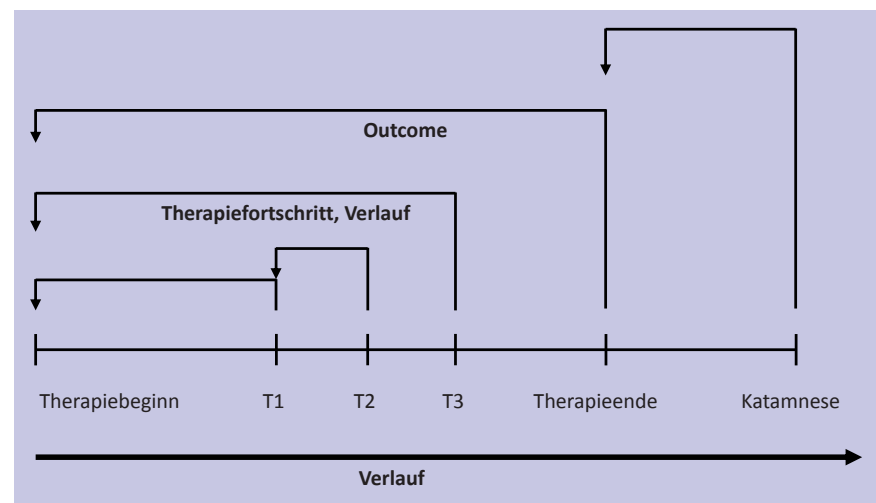

Abb. 2. Zeitraster möglicher Messabstände (T) in der Psychotherapie.

\section{Psychometrische Verfahren}

Allgemein lassen sich Ad-hoc-Verfahren von psychometrischen Verfahren unterscheiden. Ad-hoc-Verfahren werden meist ohne hinreichende testtheoretische Absicherung entwickelt. Während dies früher in Forschung und Praxis häufiger der Fall war, finden Ad-hoc-Verfahren in Anbetracht der Vielzahl verfügbarer Alternativen heute zunehmend weniger Anwendung. Lediglich für sehr spezielle Fragestellungen, für die keine Verfahren existieren, ist dieses Vorgehen heute noch legitim. Von psychometrischen Verfahren soll dann gesprochen werden, wenn sie basierend auf einer Testtheorie (klassisch oder probabilistisch) entwickelt wurden und bestimmten psychometrischen Kriterien genügen. Betrachtet man die vorliegenden Verfahren, so sind diese bis heute (trotz methodischer Vorteile der probabilistischen Testtheorie, vgl. z.B. Stieglitz [2008]) fast ausschließlich an der klassischen Testtheorie orientiert. Bezogen auf diese sind die auf Lienert [1969] zurückgehenden Haupt- und Nebengütekriterien (zum Teil auch als psychometrische und anwendungsbezogene Evaluationskriterien bezeichnet [Stieglitz, 2012]) von Bedeutung. 
Zu den Hauptgütekriterien gehören Objektivität, Reliabilität und Validität, die sich jeweils noch weiter differenzieren lassen; zu den Nebengütekriterien Normierung, Vergleichbarkeit, Ökonomie und Nützlichkeit. Nicht alle lassen sich quantifizieren wie z.B. Reliabilität und Validität. Zudem sind nicht alle Kriterien gleichgewichtig bezogen auf ein bestimmtes Instrument und dessen Anwendung in Hinblick auf ein bestimmtes Assessment-Ziel.

Bezogen auf die Psychotherapie und die Anwendung von psychometrischen Verfahren kann man davon ausgehen, dass möglichst alle Kriterien hinreichend erfüllt sein müssen. Eine besondere Rolle kommt unter dem Praxisaspekt den Nebengütekriterien zu. Da es z.B. um die Quantifizierung von klinischen Merkmalen geht, müssen Normen oder zumindest Cut-off-Werte existieren. Die Nützlichkeit muss belegt sein, da die Informationen für die Therapie konkret genutzt werden sollen. Aufgrund von Zeitfragen muss ein Verfahren auch ökonomisch in der Durchführung sein.

Von besonderer Bedeutung in der Psychotherapie ist der Einsatz von Verfahren zur Veränderungsmessung [vgl. im Überblick Stieglitz und Hiller, 2014]. Nach Stieglitz und Baumann [2001] lassen sich hier verschiedene Vorgehensweisen unterscheiden:

- Indirekte Veränderungsdiagnostik

- Direkte Veränderungsdiagnostik

- Beurteilung des (psychopathologischen) Status nach Therapieende

- Beurteilung der Therapiezielverwirklichung.

Während Letzteres einen eigenständigen Ansatz darstellt, der auf individuelle Therapiezieldefinitionen und deren Erreichung basiert [Watzke et al., 2014], kommen bei den anderen 3 Ansätzen psychometrische Verfahren zum Einsatz. Bei der indirekten Veränderungsdiagnostik werden Veränderungsinformationen z.B. durch Differenzwertbildungen zwischen 2 Messzeitpunkten erhoben (z.B. Therapieanfang und -ende). Bei der Bewertung von Veränderungen findet sich in der Literatur eine Vielzahl von einfachen (z.B. $50 \%$ Reduktion gegenüber dem Ausgangswert) bis hin zu komplexen (z.B. klinisch signifikante Veränderungen) Vorschlägen [Stieglitz, 2012; Moessner und Kordy, 2014; Kirchmann, 2013].

Einen eigenständigen Ansatz stellt die direkte Veränderungsdiagnostik dar. Dabei muss eine Person eine stattgefundene Veränderung direkt gegenüber einem definierten Bezugspunkt (z.B. Therapiebeginn) einstufen. Für diesen klinisch interessanten, methodisch aber nicht ganz unproblematischen Zugang [vgl. Stieglitz, 2008] gibt es bisher nur wenige Instrumente (z.B. Veränderungsfragebogen des Erlebens und Verhaltens (VEV) von Zielke und Kopf-Mehnert [1978]). Bei der Statusbestimmung nach Therapieende müssen z.B. Normen oder Cut-off-Werte vorliegen, um einschätzen zu können, inwieweit eine Person nach Therapieende z.B. im Bereich des Gesunden liegt. Der direkten Veränderungsdiagnostik und der Statusbestimmung nach Therapieende ist gemeinsam, dass nur ein Messzeitpunkt notwendig ist.

Gleich welche Art der Veränderungsbeurteilung gewählt wird, besteht der erste Schritt in der Auswahl und Festlegung auf ein oder mehrere Instrumente. Aufgrund der mittlerweile langen Tradition der Anwendung und Entwicklung von Verfahren auch für die Psychotherapie, ist es dem Nutzer kaum noch möglich, sich selbst einen hinreichend guten Überblick zu verschaffen. Hierzu stehen ihm jedoch mittlerweile eine Reihe von hilfreichen Übersichten zur Verfügung [z.B. Stieglitz et al., 2001; CIPS, 2005]. Be- sonders zu erwähnen sind die Zusammenstellungen von Brähler et al. [2003] und Schumacher et al. [2003] bzw. Strauß und Schumacher [2005]. Bei den systematischen Darstellungen einer Vielzahl von Verfahren wird zum Teil explizit Bezug zur Psychotherapie genommen.

Ein Defizit bei der Auswahl und Anwendung von Verfahren besteht jedoch darin, dass es bis heute kein «evidence-based assessment» [vgl. im Überblick Hunsley und Mash, 2007] mit Vorgaben gibt, welche verbindlichen Anforderungen an Verfahren gestellt werden sollten und welche Verfahren zur Anwendung kommen sollten. Leitlinien verschiedener nationaler wie internationaler Fachgesellschaften bezüglich einzelner Störungsbilder fokussieren fast ausschließlich auf die Therapie; eine psychometrische Diagnostik wird (wenn überhaupt) eher am Rande erwähnt [Freyberger und Stieglitz, 2006] und bleibt oft allgemein in Hinblick auf die Empfehlung einzelner Verfahren.

Der Anwender muss sich daher bei der Auswahl eines Verfahrens auf die oben genannten psychometrischen Kriterien beziehen (speziell Nebengütekriterien) oder sich auf Empfehlungen verlassen. Zur allgemeinen Orientierung bei der Auswahl von Verfahren sind nach Rief et al. [2006; vgl. auch Stieglitz, 2008] unter anderem folgende Aspekte wichtig bzw. Fragen zu beantworten:

- Ist der Anwendungsbereich adäquat?

- Sind die Testgütekriterien erfüllt?

- Ist der Zeitbezug adäquat?

- Ist die eigene Qualifikation zur Anwendung vorhanden?

- Sind die Handhabung und der Aufwand adäquat?

- Ist das Instrument hinreichend verbreitet?

Speziell der Aspekt der eigenen Qualifikation wird leider oft zu wenig berücksichtigt. Legt man strenge Kriterien an, sind als allgemeine Voraussetzung hinreichende psychometrische Kenntnisse notwendig (z.B. bezüglich der Bewertung der Qualität eines Verfahrens). Die Kenntnisse in der Anwendung sind ein weiterer wichtiger Aspekt. Bei Fremdbeurteilungsverfahren ist die adäquate Ausbildung (z.B. durch Trainings) die unabdingbar notwendige Voraussetzung für die adäquate Anwendung. Dies ist leider bis heute ein großes Problem. Immer wieder werden Instrumente ohne eine entsprechende Qualifikation eingesetzt.

Im Hinblick auf die Psychotherapie (speziell in der Forschung) wird immer wieder gefordert, der Komplexität des Patienten und seiner Problematik dadurch gerecht zu werden, indem ein multimodaler Ansatz zur Anwendung kommt [Baumann und Stieglitz, 2001]. Innerhalb dieses Ansatzes wird zwischen unterschiedlichen Datenebenen, Datenquellen und Funktionsbereichen unterschieden. In Studien ist dies durchaus denkbar; für die Praxis jedoch scheint dieser Ansatz eher unrealistisch zu sein (unter anderem aus Zeitgründen). Während auf Ebene der Funktionsbereiche verschiedene Aspekte erfassbar sind (z.B. Depressivität und Ängstlichkeit), spielen auf der Ebene der Datenquellen vor allem der Patient und unabhängige Rater die zentrale Rolle. Die verwendeten wichtigsten Instrumentengruppen sind die Selbst- und Fremdbeurteilungsverfahren (Tab. 3; 4). Bei Selbstbeurteilungsverfahren ist der Patient die alleinige Informationsquelle, die einer Einschätzung zugrunde liegt, während bei der Fremdbeurteilung zusätzlich Beobachtungen des Therapeuten und Informationen über den Patienten sowie dessen Aussagen über sich selbst in die Bewertung einfließen. Beide Verfahrensgruppen sind im klinischen Kontext, d.h. auch in der Psychotherapie, die wichtigsten und am häufigsten 
Tab. 3. Unterschiede zwischen Selbst- und Fremdbeurteilungsverfahren

\begin{tabular}{lll}
\hline & Selbstbeurteilungsverfahren & Fremdbeurteilungsverfahren \\
\hline Vorteile & zeitökonomisch; meist Normen vorhanden & änderungssensitiver; differenzieren besser zwischen verschiedenen \\
Nachteile & $\begin{array}{l}\text { Fei bis zu mittleren Störungsgraden einsetzbar; } \\
\text { kognitive Mindestanforderungen }\end{array}$ & zeitaufwendig; (kontinuierliches) Training notwendig \\
\hline
\end{tabular}

eingesetzten Verfahrensgruppen, zumal sie auch zahlenmäßig die größten Instrumentengruppen darstellen. Neben einer Reihe von Gemeinsamkeiten (unter anderem breiter Anwendungsbereich), weisen sowohl die Selbst- als auch die Fremdbeurteilungsverfahren spezifische Vor- und Nachteile auf, wie sie in Tabelle 3 zusammengefasst sind. Häufig wird die Frage gestellt, ob ein Selbst- oder ein Fremdbeurteilungsverfahren zum Einsatz kommen soll. Dies kann beim heutigen Wissensstand nur dahingehend beantwortet werden, dass sie sich nicht gegenseitig ersetzen können, da sie unterschiedliche Perspektiven darstellen. Ihnen kommt somit eher eine komplementäre Funktion zu [Baumann und Stieglitz, 2001; Stieglitz, 2008].

Nachfolgend soll daher ein Überblick zu Selbst- und Fremdbeurteilungsverfahren gegeben werden, die in der Psychotherapie Anwendung finden können, wobei aufgrund der Fülle existierender Verfahren eine Auswahl anhand folgender Kriterien getroffen werden musste:

- Outcome-Instrumente (keine Prozessinstrumente; vgl. hierzu Stieglitz und Hiller [2014])

- Beschränkung auf ausgewählte Störungsbereiche

- Verfahren, für die zumindest deutschsprachige Übersetzungen vorliegen

- häufig eingesetzte Verfahren (wenn möglich Goldstandards)

- wenn möglich, mindestens jeweils ein Selbst- und ein Fremdbeurteilungsverfahren (zu anderen Verfahrensgruppen in der Klinischen Psychologie und Psychotherapie; vgl. Stieglitz et al. [2001])

- Cut-off-Werte, besser noch Normwerte, mindestens jedoch Referenzwerte.

\section{Störungsübergreifende Verfahren}

Störungsübergreifende Verfahren sind dadurch gekennzeichnet, dass sie bei (fast) allen Störungsgruppen einsetzbar sind. Je nach erfasstem Konstrukt liefern sie unterschiedliche Informationen zu vielfältigen Facetten, die über das eigentlich jeweils im Vordergrund stehende Störungsbild hinausgehen, und stellen somit eine Ergänzung der störungsbezogenen Diagnostik dar. Sie ermöglichen zudem eine Vergleichbarkeit zwischen Patienten unterschiedlicher Störungsgruppen. Dies ist insbesondere bei der Anwendung im Rahmen von Testbatterien z.B. im Kontext der Qualitätssicherung sinnvoll [vgl. z.B. Hiller et al., 2011]. Ein wichtiger Nachteil besteht jedoch darin, dass störungsübergreifende Verfahren bei alleiniger Anwendung oft zu unspezifisch und zu ungenau sind. In Tabelle 2 sind exemplarisch häufig, auch im Bereich der Psychotherapie eingesetzte Verfahren aufgenommen [vgl. auch Brähler et al., 2003].

Im Vergleich zu störungsbezogenen Verfahren ist die Zahl deutlich geringer, speziell was die allgemeine Psychopathologie betrifft. Dies trifft vor allem Fremdbeurteilungsverfahren zu.
Hier findet heute nur noch das AMDP-System Anwendung, was jedoch ursprünglich im Kontext der Psychopharmakologie entwickelt wurde. Auf der Ebene der Selbstbeurteilungsverfahren kommt am häufigsten die SCL-90 [Franke, 2013] zum Einsatz, die jedoch unter methodischem Aspekt nicht unproblematisch ist (z.B. aufgrund der postulierten Mehrdimensionalität; vgl. Glöckner-Rist und Stieglitz [2011]). Die Globalwerte sind jedoch als valide Indikatoren der Gesamtbeeinträchtigungen interpretierbar. Zunehmend häufiger zum Einsatz kommt der Outcome Questionnaire [OQ-45.2; Lambert et al., 2002], der einen stärkeren Bezug zur Psychotherapie hat, da er in diesem Kontext auch entwickelt wurde. Er ermöglicht zusätzlich zur Quantifizierung der Symptomatologie auch die Erfassung zwischenmenschlicher Beziehungen sowie sozialer Integration als therapierelevante Dimensionen. Verschiedene Validitätsstudien unterstreichen speziell auch die Eignung des Instruments zur Veränderungserfassung [z.B. Vermeersch et al., 2000; Haug et al., 2004]. Jedoch auch die Erfassung anderer störungsübergreifender Bereiche ist wichtig wie z.B. allgemeiner Beschwerden oder auch der stärker fluktuierenden Befindlichkeiten oder Stimmungen. Hierfür liegt eine Reihe bewährter Verfahren vor. Neben den aufgeführten Bereichen sind weitere wichtig, die jedoch außerhalb des Rahmens dieser Übersicht liegen wie z.B. Beeinträchtigungen oder Lebensqualität [vgl. hierzu im Überblick Stieglitz et al., 2001; Brähler et al., 2003; Schumacher et al., 2003; Bengel et al., 2008].

\section{Störungsbezogene Verfahren}

Störungsbezogene, manchmal auch als störungsgruppenbezogene oder störungsspezifische Verfahren bezeichnet, fokussieren, wie der Name schon nahelegt, primär auf eine einzelne Störungsgruppe. Eine weitere Differenzierung ist möglich, inwieweit sie einen Therapiebezug haben oder nicht, d.h. aus dem theoretischen Therapiemodell abgeleitet sind. Letzteres ist jedoch eher selten der Fall (Ausnahme: Verfahren aus der kognitiven Therapie nach Beck).

Ein wesentlicher Vorteil störungsbezogener Verfahren besteht darin, dass die jeweils erfassten Informationen einen direkten Bezug zur Störung haben und damit spezifische Informationen liefern. Nachteilig ist, dass die meist eindimensionalen Verfahren nur einen Symptombereich abdecken, sodass andere klinisch relevante Facetten verloren gehen und daher oft eine Ergänzung durch störungsübergreifende Verfahren notwendig ist (Tab. 2).

In Tabelle 4 finden sich die wichtigsten Verfahren für ausgewählte Störungsgruppen, die in der Psychotherapie von besonderer Relevanz sind. Eine umfassende Zusammenstellung von Verfahren z.B. für Persönlichkeitsstörungen findet sich in der Monografie von Antony und Barlow [2011; vgl. auch Strupp et al., 1997]. Gerade der Bereich der Persönlichkeitsstörungen ist eher noch als defizitär zu bezeichnen, da es kaum allgemeine Verfahren zur Therapieevaluation gibt. Positive Entwicklungen sind al- 
Tab. 4. Auswahl von Skalen zur störungsbezogenen Diagnostik

\begin{tabular}{|c|c|c|c|c|}
\hline Bereich & Verfahren & $\mathrm{S} / \mathrm{F}$ & Kennzeichen & Kommentar \\
\hline \multirow[t]{7}{*}{$\begin{array}{l}\text { Affektive } \\
\text { Störungen }\end{array}$} & $\begin{array}{l}\text { Beck-Depressions-Inventar } \\
\text { [BDI-II; Beck et al., 2006] }\end{array}$ & $\mathrm{S}$ & 21 Items, Gesamtwert & orientiert am DSM-IV \\
\hline & $\begin{array}{l}\text { Major Depression (ICD-10) Inventory } \\
\text { [MDI; Beck et al., 2001] }\end{array}$ & $\mathrm{S}$ & 10 Items, Gesamtwert & $\begin{array}{l}\text { dimensionale und kategoriale } \\
\text { Auswertung }\end{array}$ \\
\hline & $\begin{array}{l}\text { Hamilton-Depressions-Skala } \\
\text { [HAMD; CIPS, 2005] }\end{array}$ & $\mathrm{F}$ & 21 Items, Gesamtwert & $\begin{array}{l}\text { am häufigsten eingesetzte } \\
\text { Depressionsskala; methodisch } \\
\text { problematisch }\end{array}$ \\
\hline & $\begin{array}{l}\text { Montgomery-Åsberg Depression Rating } \\
\text { Scale [MADRS; CIPS, 2005] }\end{array}$ & $\mathrm{F}$ & 10 Items, Gesamtwert & $\begin{array}{l}\text { HAMD psychometrisch } \\
\text { überlegen }\end{array}$ \\
\hline & $\begin{array}{l}\text { Bech-Rafaelsen-Depressions-Skala } \\
\text { [BRMS; Stieglitz et al., 1998] }\end{array}$ & $\mathrm{F}$ & 11 Items, Gesamtwert & $\begin{array}{l}\text { HAMD psychometrisch } \\
\text { überlegen }\end{array}$ \\
\hline & $\begin{array}{l}\text { Manie-Selbstbeurteilungs-Skala } \\
\text { [MSS; Krüger et al., 1998] }\end{array}$ & $\mathrm{S}$ & 48 Items, Gesamtwert & $\begin{array}{l}\text { bei ausgeprägter manischer } \\
\text { Symptomatik problematisch }\end{array}$ \\
\hline & $\begin{array}{l}\text { Bech-Rafaelsen-Manie-Skala } \\
\text { [BRMS; CIPS, 2005] }\end{array}$ & $\mathrm{F}$ & 11 Items, Gesamtwert & $\begin{array}{l}\text { auch bei schwerer Symptomatik } \\
\text { einsetzbar }\end{array}$ \\
\hline \multirow[t]{2}{*}{$\begin{array}{l}\text { Panik und } \\
\text { Agoraphobie }\end{array}$} & $\begin{array}{l}\text { Panik- und Agoraphobie-Skala } \\
\text { [PAS; Bandelow, 1997] }\end{array}$ & $\mathrm{S}+\mathrm{F}$ & $\begin{array}{l}\text { je } 13 \text { Items, } 5 \text { Subskalen, } \\
\text { Gesamtwert }\end{array}$ & $\begin{array}{l}\text { Selbst- und } \\
\text { Fremdbeurteilungsversion }\end{array}$ \\
\hline & $\begin{array}{l}\text { Fragebogen zu körperbezogenen Ängsten, } \\
\text { Kognitionen und Vermeidung } \\
\text { [AKV; Ehlers et al., 2001] }\end{array}$ & $\mathrm{S}$ & 61 Items, 3 Subskalen & $\begin{array}{l}\text { Normwerte für verschiedene } \\
\text { klinische Gruppen und Gesunde }\end{array}$ \\
\hline \multirow[t]{2}{*}{ Zwangsstörungen } & $\begin{array}{l}\text { Yale-Brown Obsessive Compulsive Scale } \\
\text { [Y-BOCS; Büttner-Westphal et al., 1991] }\end{array}$ & $\mathrm{F}$ & $\begin{array}{l}18 \text { Items, Gesamtwert, } \\
2 \text { Subskalen }\end{array}$ & Interviewleitfaden vorhanden \\
\hline & $\begin{array}{l}\text { Obsessive-Compulsive Inventoy-Revised } \\
\text { [OCI-R; Foa et al., 2009] }\end{array}$ & $\mathrm{S}$ & $\begin{array}{l}18 \text { Items, } 5 \text { Subskalen, } \\
\text { Gesamtwert }\end{array}$ & kurze Durchführungszeit \\
\hline \multirow[t]{2}{*}{$\begin{array}{l}\text { Posttraumatische } \\
\text { Belastungsstörung }\end{array}$} & $\begin{array}{l}\text { Posttraumatic Symptom Scale (PSS) bzw. } \\
\text { Posttraumatic Diagnostic Scale } \\
\text { [PDS; Stieglitz, 2003] }\end{array}$ & $\mathrm{S}$ & $\begin{array}{l}17 \text { DSM-IV-Kriterien, } \\
\text { Gesamtwert }\end{array}$ & $\begin{array}{l}\text { dimensionale und kategoriale } \\
\text { Auswertung }\end{array}$ \\
\hline & $\begin{array}{l}\text { Clinician-Administered PTSD Scale } \\
\text { [CAPS; Nyberg und Stieglitz, 2005] }\end{array}$ & $\mathrm{F}$ & $\begin{array}{l}17 \text { DSM-Kriterien, } \\
3 \text { Subskalen, Gesamtwert }\end{array}$ & $\begin{array}{l}\text { viele ergänzende Informationen } \\
\text { über Symptomatik hinaus }\end{array}$ \\
\hline \multirow[t]{2}{*}{ Dissoziative Störungen } & $\begin{array}{l}\text { Fragebogen zu Dissoziativen Symptomen } \\
\text { [FDS; Spitzer et al., 2005] }\end{array}$ & $\mathrm{S}$ & $\begin{array}{l}44 \text { Items, } 4 \text { Subskalen, } \\
\text { Gesamtwert }\end{array}$ & $\begin{array}{l}\text { verschiedene } \\
\text { Auswertungsmöglichkeiten; } \\
\text { Kurz- und Screeningversion } \\
\text { vorhanden }\end{array}$ \\
\hline & $\begin{array}{l}\text { Heidelberger Dissoziationsinventar } \\
\text { [HDI; Brunner et al., 2008] }\end{array}$ & $\mathrm{S}+\mathrm{F}$ & $\begin{array}{l}28 \text { Items } \\
\text { (Erwachsenenversion), } \\
30 \text { Items } \\
\text { (Jugendlichenversion) }\end{array}$ & $\begin{array}{l}\text { Selbst- und } \\
\text { Fremdbeurteilungsversion; } \\
\text { Erwachsenen und Jugendlichen- } \\
\text { version; dimensionale und } \\
\text { kategoriale Auswertung }\end{array}$ \\
\hline
\end{tabular}

lein im Kontext der Borderline-Persönlichkeitsstörung erkennbar [z.B. Bohus et al., 2001]. Beispiele von Verfahren zur Schizophrenie, zu Somatoformen Störungen und zu Störungen durch Psychotrope Substanzen finden sich bei Stieglitz et al. [2001].

Spezifische diagnostische Verfahren finden sich zudem in den einzelnen störungsbezogenen Bänden der bereits erwähnten Reihe «Fortschritte der Psychotherapie» sowie der Reihe «Evidenzbasierte Leitlinien der Psychotherapie» des Hogrefe-Verlags und den Therapiemanualen des Beltz-Verlags.

Auf einige allgemeine und zentrale Aspekte der störungsbezogenen Diagnostik sei nachfolgend noch kurz eingegangen. Die Zunahme an immer weiter entwickelten Skalen führt dazu, dass zwischenzeitlich für viele Bereiche konkurrierende Verfahren vorliegen. Dies ist besonders evident im Bereich der Depression, für die mittlerweile mehr als 100 Instrumente existieren [Stieglitz, 2008].
Dies ist sicherlich als problematisch anzusehen, da es nach wie vor keine Einigung darüber gibt, was die konstituierenden Merkmale des sogenannten Depressiven Syndroms sind. Jede Skala deckt unterschiedliche Facetten ab [Stieglitz, 2008], sodass selbst bei ähnlicher Bezeichnung die Inhalte der einzelnen Skalen sehr unterschiedlich sein können. Am deutlichsten wird dies beim Vergleich der beiden am häufigsten eingesetzten Verfahren, der HamiltonDepressionsskala [HAMD; CIPS, 2005] und dem Beck-Depressions-Inventar [BDI; Beck et al., 2006]. Während bei Ersterem der Fokus eher auf körperlichen Symptomen liegt, erfasst das BDI die kognitive Symptomatik. Andere Depressionsskalen fokussieren wiederum stärker auf affektive Symptome oder Antrieb [vgl. Stieglitz, 2008]. Dies gilt es bei der Auswahl und der Interpretation unbedingt zu bedenken. Bezüglich der HAMD ist zusätzlich zu erwähnen, dass die Skala zwischenzeitlich den Status eines Goldstan- 
Tab. 5. Ratingskalen im Rahmen der Angstdiagnostik nach Hoyer und Margraf [2003]; siehe auch Tabelle 4

\begin{tabular}{|c|c|c|}
\hline Bereich & $\begin{array}{l}\text { Anzahl } \\
\text { Verfahren }\end{array}$ & Beispiele \\
\hline $\begin{array}{l}\text { Messung von Angst, Ängstlichkeit } \\
\text { und Angststörungen }\end{array}$ & 20 & Beck-Angstinventar (BAI), State-Trait-Angstinventar (STAI) \\
\hline Agoraphobie und Panikstörung & 8 & $\begin{array}{l}\text { Fragebogen zu körperbezogenen Ängsten, Kognitionen und } \\
\text { Vermeidung (AKV) } \\
\text { Panik- und Agoraphobieskala (PAS) }\end{array}$ \\
\hline Generalisierte Angststörung & 7 & Anxious Thoughts Inventory (AnTI) \\
\hline Soziale Phobie, soziale Ängste & 21 & $\begin{array}{l}\text { Liebowitz-Soziale-Angst-Skala (LSAS), Soziale Phobie- und } \\
\text { Angst-Inventar (SPAI) }\end{array}$ \\
\hline Zwangsstörung & 14 & $\begin{array}{l}\text { Hamburger Zwangsinventar (HZI), Yale-Brown-Obsessive- } \\
\text { Compulsive Scale (Y-BOCS) }\end{array}$ \\
\hline Posttraumatische Belastungsstörung & 9 & $\begin{array}{l}\text { Posttraumatic Stress Diagnostic Scale (PSDS), } \\
\text { Clinician-Administered PTSD Scale (CAPS) }\end{array}$ \\
\hline $\begin{array}{l}\text { Spezifische Phobien, Furcht vor } \\
\text { spezifischen Situationen, } \\
\text { Gesundheits- und Todesangst }\end{array}$ & 29 & Fear Survey Schedule (FSS), Spinnenphobie-Fragebogen (SPF) \\
\hline $\begin{array}{l}\text { Angstdiagnostik bei Kindern und } \\
\text { Jugendlichen }\end{array}$ & 18 & $\begin{array}{l}\text { Angstfragebogen für Kinder (AFS), Child PTSD Symptom } \\
\text { Scale (CPSS) }\end{array}$ \\
\hline
\end{tabular}

dards erreicht hat, was sich dadurch belegen lässt, dass die Skala in (fast) jeder Depressionsstudie eingesetzt und in Therapiestudien meist als Haupt-Outcome gewählt wird. Dies ist umso verwunderlicher, da seit Jahren die methodischen Schwächen der Skala hinreichend bekannt [Stieglitz, 2008] und bessere Alternativen vorhanden sind, die zudem durch Kürze überzeugen (unter anderem Montgomery-Åsberg-Depressions-Skala, MADRS; CIPS [2005] sowie die Bech-Rafaelsen-Melancholie-Skala, BRMS; Stieglitz et al. [1998]). Ist man neben der globalen Einschätzung des Depressiven Syndroms auch an einer differenzierten Betrachtung der verschiedenen Teilkomponenten interessiert, so liefert z.B. der Differentielle Depressions-Fragebogen [DDF; Steck, 1998] mit seinen 6 verschiedenen Subsyndromen ergänzende Informationen (unter anderem phobisch-vegetativ, paranoid-depressiv).

Eine Entwicklungstendenz geht in Richtung einer zunehmenden Ausdifferenzierung bestimmter Bereiche. Exemplarisch genannt sei der Angstbereich. In Tabelle 5 sind einige Instrumente bezogen auf Subgruppen von Angststörungen aufgelistet. Während vor ca. 30-40 Jahren nur das State-Trait Anxiety Inventory [STAI; CIPS, 1977] existierte, kann der Anwender heute auf eine weite Palette von Verfahren zu verschiedenen Subtypen von Angststörungen zurückgreifen. Für andere Störungsbereiche wie beispielsweise Dissoziative Störungen sind ähnliche Entwicklungen erkennbar [z.B. Spitzer, 2004].

Eine weitere Entwicklungstendenz betrifft die zunehmende Verbindung von kategorialer und dimensionaler Diagnostik [Stieglitz, 2008]. Die kategoriale Diagnostik, deren Ergebnis die Diagnosen eines Klassifikationssystems sind, steht meist am Anfang des diagnostischen Prozesses (Abb. 1). Sie ist jedoch im Hinblick auf die Therapieplanung und -evaluation nur bedingt hilfreich. Es bedarf der Ergänzung um psychometrische Verfahren. Diese zeigen jedoch oft keinen Bezug zur Operationalisierung einer Störung in einem Klassifikationssystem, da sie meist älter sind als die aktuellen Systeme. Hier ist jedoch allmählich eine Veränderung in Richtung einer Kombination beider Sichtweisen erkennbar. So zeigen sich z.B. im Bereich der Depression und

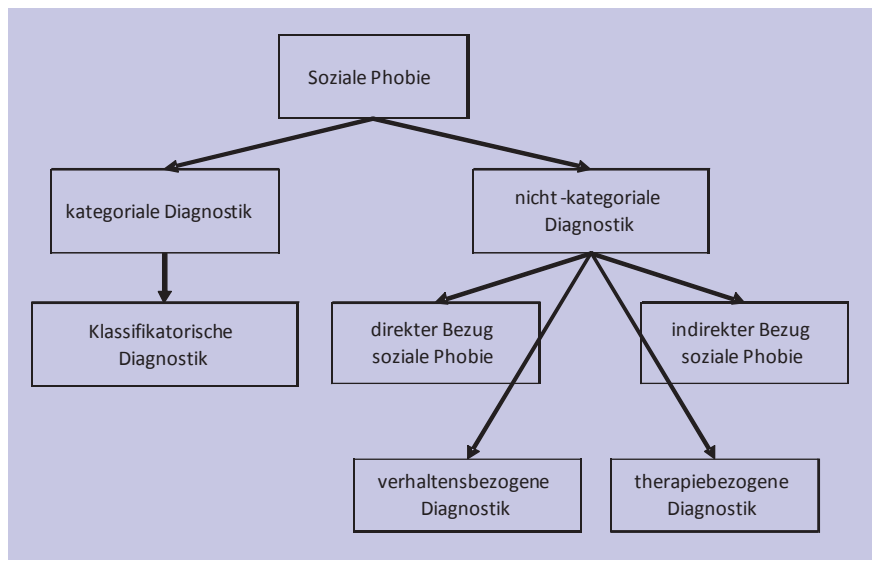

Abb. 3. Störungsbezogene Diagnostik am Beispiel der Sozialen Phobie [vgl. auch Stangier et al., 2009].

PTBS Entwicklungen von Selbstbeurteilungsverfahren, die sich auf die diagnostischen Kriterien der Störungskategorien beziehen (Tab. 4). So wurden z.B. im Major Depression Inventory [MDI; Bech et al., 2001] die Kriterien der Depressiven Episode der ICD-10 zu Fragen umformuliert. Psychometrische Analysen dieses Selbstbeurteilungsverfahrens sind als vielversprechend $\mathrm{zu}$ bewerten [z.B. Olson et al., 2003; Konstantinidis et al., 2011]. Die Skala ermöglicht einerseits die Quantifizierung der Symptomatik hinsichtlich ihrer Stärke (Bezugsrahmen letzte 2 Wochen) und liefert andererseits mit der Orientierung an der Operationalisierung der depressiven Episode nach ICD-10 Hinweise auf eine kategoriale Interpretation. Abschließend sei auf eine besonders für die Therapie relevante Entwicklung hingewiesen, nämlich die Ausdifferenzierung der nicht-kategorialen Diagnostik über die Syndrom-Ebene hinaus. In Abbildung 3 sowie Tabelle 6 ist dies am Beispiel der Sozialen Phobie dargestellt.

Bezüglich der nicht-kategorialen Diagnostik lassen sich verschiedene Bereiche differenzieren, die für die Therapie sinnvoll 
Tab. 6. Verfahren im Kontext der Sozialen Phobie. Nähere Angaben zu den Verfahren siehe Stangier et al. [2009] sowie Heidenreich und Stangier [2002]

\begin{tabular}{ll}
\hline Ebene & Verfahren \\
\hline Direkter Bezug zur Störung & Social Phobia and Anxiety Inventory (SPAI) \\
& Liebowitz-Soziale-Angst-Skala (LSAS) \\
Indirekter Bezug zur Störung & Interaktions-Angst-Fragebogen (IAF) \\
& Skala «Unsicherheit im Sozialkontakt» der Symptom- \\
& Checklist (SCL-90-R) \\
Therapiebezogene Diagnostik & Fragebogen zu sozialphobischen Kognitionen (SPK) \\
& Fragebogen zu sozialphobischem Verhalten (SPV) \\
Verhaltensbezogene Diagnostik & Rollenspiele, Verhaltensbeobachtungen \\
\hline
\end{tabular}

sind (z.B. Planung, Evaluation). Bis auf die verhaltensbezogene Diagnostik sind psychometrische Verfahren in allen Diagnostikbereichen verfügbar. Dies betrifft Verfahren zur Evaluation der Symptomatik im Querschnitt wie im Verlauf sowie Verfahren, die direkt in der Therapieplanung (z.B. Erfassung spezifischer Kognitionen) eingesetzt werden können. Derartige Konzeptualisierungen wären auch für andere Störungsbereiche sehr wünschenswert.

\section{Fazit und Ausblick}

Eine therapiebegleitende Diagnostik spielt trotz vielfältiger Verfahrensentwicklungen in den letzten Jahrzehnten leider immer noch nicht die zentrale Rolle, die ihr zusteht. Die zahlreichen Möglichkeiten werden immer noch zu wenig genutzt, sind aber leider vielfach auch gar nicht bekannt. Entscheidend für eine weitere Verbreitung wird sein, den therapeutischen Nutzen für die Arbeit zu erkennen. Zu den Minimalanforderungen sollte die Anwendung mindestens eines Verfahrens zu Therapiebeginn und -ende sein, besser noch die wiederholte Anwendung im Therapieverlauf, um Fehlentwicklungen rechtzeitig zu erkennen [vgl. z.B. Grawe und Braun, 1994]. Unter ökonomischen Gesichtspunkten sollten speziell auch die zunehmenden Angebote einer computerisierten Nutzung stärker in Anspruch genommen werden.

Auch wenn die prinzipiellen Möglichkeiten der Anwendung psychometrischer Verfahren insgesamt als positiv anzusehen sind, gibt es eine Reihe von Defiziten, deren Bewältigung ein weiterer Schritt zur Verbesserung einer therapiebegleitenden Diagnostik sein wird. Zu diesen Defiziten zählen:

- Mangel an Verfahren mit einem direkten Bezug zur jeweiligen Therapiemethode

- Mangel an Verfahren mit Orientierung an den gültigen Klassifikationssystemen

- expliziter Mangel an Verfahren in bestimmten Störungsbereichen (unter anderem Persönlichkeitsstörungen)
- Mangel an Kurzskalen mit hinreichender psychometrischer Qualität zur wiederholten Anwendung innerhalb der Therapie

- Mangel an Berücksichtigung von Aspekten der Änderungssensitivität bereits bei der Testentwicklung bzw. bei der nachträglichen Überprüfung der Eignung für die Verlaufsmessung [vgl. hierzu auch Stieglitz und Hiller, 2014].

Insgesamt wäre es wünschenswert, auch im Bereich der therapiebegleitenden Diagnostik Standards im Sinne eines «evidencebased assessment» zu etablieren. Begrüßenswert wären auch Qualitätsstandards, wie sie mit der DIN-Norm 33430 für die berufsbezogene Eignungsbeurteilung vorgelegt wurden [Kersting, 2008]. Leider sind derartige Entwicklungen im Bereich der Klinischen Psychologie und Psychotherapie noch nicht erkennbar. Im deutschsprachigen Bereich wäre bei der Überarbeitung existierender Therapieleitlinien auch eine differenziertere Darstellung mit spezifischen Aussagen zu folgenden diagnostischen Aspekten notwendig [vgl. Freyberger und Stieglitz, 2006]:

- Instrumente (unter anderem die Art der Instrumente, die bei bestimmten Störungen einzusetzen sind; Anforderungen an ihre psychometrische Qualität)

- Zeitperspektive (Art der Instrumente, die zu bestimmten Zeitpunkten und mit bestimmten Assessment-Zielen einzusetzen sind)

- Auswertung (Art und Weise, wie Veränderungen bestimmt und interpretiert werden)

- Anwender (Qualifikationsanforderungen an die Anwender von Instrumenten).

\section{Disclosure Statement}

Der Autor erklärt hiermit, dass keinerlei Interessenskonflikte in Bezug auf dieses Manuskript bestehen.

\section{Literatur}

Angst J, Bech P, Boyer P, Bruinvels J, Engel R, Helmchen H, Hippius H, Lingjaerde O, Racagni G, Saletu B, Sedvall G, Silverstone JT, Stefanis CN, Stoll $\mathrm{K}$, Woggon, B: Consensus conference on the methodology of clinical trials of antidepressants, Zurich, March 1988: report of the consensus committee. Pharmacopsychiatry 1989;22:3-7.
Antony DH, Barlow MM (eds): Handbook of Assessment and Treatment Planning. New York, Guilford, 2011.

Arbeitsgemeinschaft für Methodik und Dokumentation in der Psychiatrie (AMDP): Das AMDP-System, ed. 8. Göttingen, Hogrefe, 2007.

Bandelow B: Panik- und Agoraphobie-Skala (PAS). Göttingen, Hogrefe, 1997.
Bandelow B, Margraf J: Empfehlungen für die Verwendung von Meßinstrumenten in der klinischen Angstforschung. Fortschr Neurol Psychiatr 1994; 62:361-365.

Baumann U, Stieglitz RD: Testmanual zum AMDPSystem. Empirische Studien zur Psychopathologie. Berlin, Springer, 1983. 
Baumann U, Stieglitz RD: Psychodiagnostik psychischer Störungen: Allgemeine Grundlagen; in Stieglitz RD, Baumann U, Freyberger HJ (eds): Psychodiagnostik in Klinischer Psychologie, Psychiatrie, Psychotherapie. Stuttgart, Thieme, 2001, pp 3-20.

Bech P, Rasmussen NA, Olsen LR, Noerholm V, Abildgaard W: The sensitivity and specificity of the Major Depression Inventory, using the Present State Examination as the index of diagnostic validity. J Affect Disord 2001;66:159-64.

Beck AT, Steer RA, Brown GK, Hautzinger M, Keller F, Kühner C: Beck-Depressions-Inventar (BDI-II) Frankfurt, Pearson, 2006.

Bengel J, Wirtz M, Zwingmann C (eds): Diagnostische Verfahren in der Rehabilitation. Göttingen, Hogrefe, 2008.

Bohus M, Limberger M, Frank U, Sender I, Gratwohl T, Stieglitz RD: Entwicklung der Borderline-Symptom-Liste. Psychother Psychosom Med Psychol 2001;51:201-211.

Brähler E, Schumacher J, Strauss B (eds): Diagnostische Verfahren in der Psychotherapie, ed 2. Göttingen, Hogrefe, 2003.

Brunner RM, Resch MF, Parzer P, Koch E: Heidelberger Dissoziationsinventar (HDI), ed 2. Frankfurt, Pearson, 2008.

Büttner-Westphal H, Hand I, Goodman W, Rasmussen S, Price L, Mazure L, Heninger G, Charney D Yale-Brown Obsessive Compulsive Scale (YBOCS). Verhaltenstherapie 1991;1:226-233.

Collegium Internationale Psychiatriae Scalarum (CIPS): Internationale Skalen für Psychiatrie. Berlin, CIPS, 1977.

Collegium Internationale Psychiatriae Scalarum (CIPS): Internationale Skalen für Psychiatrie, ed 5. Göttingen, Hogrefe, 2005.

Doering S, Hörz S (eds): Handbuch der Strukturdiagnostik. Stuttgart, Schattauer, 2012.

Ehlers A, Margraf J, Chambless D: AKV - Fragebogen zu körperbezogenen Ängsten, Kognitionen und Vermeidung, ed 2. Göttingen, Beltz Test, 2001.

Fähndrich E, Stieglitz RD: Leitfaden zur Erfassung des psychopathologischen Befundes. Halbstrukturiertes Interview anhand des AMDP-Systems, ed 3. Göttingen, Hogrefe, 2007.

Fahrenberg J: Die Freiburger Beschwerdenliste (FBL). Form FBL-G und revidierte Form FBL-R. Göttingen, Hogrefe, 1994.

Foa EB, Huppert JD, Leiberg S, Langner R, Kichic R, Hajcak G, Salkovskis PM: OCI-R. ObessessiveCompulsive Inventory-Revised. Frankfurt, Pearson, 2009.

Franke G: Symptom-Checklist-90 ${ }^{\circledR}$ (SCL-90 ${ }^{\circledR}$-S). Göttingen, Hogrefe, 2013.

Freyberger HJ, Stieglitz RD: Leitlinien zur Diagnostik in der Psychiatrie und Psychotherapie. Z Psychiatr Psychol Psychother 2006;54:23-32.

Glöckner-Rist A, Stieglitz RD: TBS-TK Rezension: SCL-90-R - Die Symptom-Checkliste von L.R. Derogatis (deutsche Version). Psychol Rundsch 2011; 63:73-75.

Grawe K, Braun U: Qualitätskontrolle in der Psychotherapiepraxis. Z Klin Psychol 1994;23:242-267.

Haug S, Puschner B, Lambert MJ, Kordy H: Veränderungsmessung in der Psychotherapie mit dem Ergebnisbogen (EB-45). Zeitschrift für Differentielle und Diagnostische Psychologie 2004;25:141-151.

Heidenreich T, Stangier U: Störungsspezifische Diagnostik der Sozialen Phobie; in Stangier U, Fydrich T (eds): Soziale Phobie und Soziale Angststörung. Göttingen, Hogrefe, 2002, pp 66-86.
Hiller W, Schindler A, Andor T, Rist F: Vorschläge zur Evaluation regulärer Psychotherapien an Hochschulambulanzen im Sinne der Phase-IVTherapieforschung. Z Klin Psychol Psychother 2011;40:22-32.

Höger D: Verfahren zur Messung des Therapieprozesses; in Eckert J, Biermann-Ratjen EM, Höger D (eds): Gesprächspsychotherapie. Berlin, Springer, 2006a, pp 273-277.

Höger D: Verfahren zur Messung des Therapieergebnisses; in Eckert J, Biermann-Ratjen EM, Höger D (eds): Gesprächspsychotherapie. Berlin, Springer, 2006b, pp 277-283.

Hoyer J, Margraf J (eds): Angstdiagnostik. Berlin, Springer, 2003.

Hunsley J, Mash EJ: Introduction to the special section on developing guidelines for the EvidenceBased Assessment (EBA) of adult disorders. Psychol Assess 2005;17:251-255.

Hunsley J, Mash EJ: Evidence-based assessment. Annu Rev Clin Psychol 2007:3:29-51.

Kersting M: Qualität in der Diagnostik und Personalauswahl - der DIN-Ansatz. Göttingen, Hogrefe, 2008.

Kirchmann H: Evaluation einzelfallbezogener Veränderungen auf kontinuierlichen Variablen. Psychother Psychosom Med Psychol 2013;63:55-57.

Konstantinidis A, Martiny K, Bech P, Kasper S: A comparison of the Major Depression Inventory (MDI) and the Beck Depression Inventory (BDI) in severly depressed patients. Int J Psychiatry Clin Pract 2011;15:56-61.

Krüger S, Bräuning P, Shugar G: Manie-Selbstbeurteilungsskala (MSS). Göttingen, Beltz Test, 1998.

Kuhl J, Kazén M: PSSI Persönlichkeits-Stil- und Störungs-Inventar, ed 2. Göttingen, Hogrefe, 2006.

Laireiter AR (ed): Diagnostik in der Psychotherapie. Wien, Springer, 2000

Lambert MJ, Hannöver W, Nissmüller K, Richard M, Kordy H: Fragebogen zum Ergebnis von Psychotherapie. Z Klin Psychol Psychother 2002;31:40-47.

Lienert GA: Testaufbau und Testanalyse. Weinheim, Beltz, 1969.

Linster HW, Härter M, Stieglitz RD (eds): Qualitätsmanagement in der Psychotherapie und Beratung. Göttingen, Hogrefe, 2003.

Lutz W (ed): Lehrbuch Psychotherapie. Bern, Huber, 2010.

Margraf J, Schneider S: Lehrbuch der Verhaltenstherapie. Band 1, ed 3. Berlin, Springer, 2009.

Meinlschmidt G, Schneider S, Margraf J (eds): Lehrbuch der Verhaltenstherapie. Band 4. Berlin, Springer, 2012.

Morfeld M, Kirchberger I, Bullinger M: SF-36 Fragebogen zum Gesundheitszustand, ed 2. Göttingen, Hogrefe, 2011.

Moessner M, Kordy H: Veränderungsmessung und Ergebnismonitoring in der klinischen Routine. Z Psychiatr Psychol Psychother 2014:im Druck.

Nyberg E, Stieglitz RD: CAPS Clinician-Administered PTSD Scale; in Strauss B, Schumacher J (eds): Klinische Interviews und Ratingskalen. Göttingen, Hogrefe, 2005, pp 64-68.

Olson LR, Jensen DV, Noerholm V, Martiny K, Bech $\mathrm{P}$ : The internal and external validity of the Major Depression Inventory in measuring severity of depressive states. Psychol Med 2003;33:351-356.

Rief W, Exner C, Martin A: Psychotherapie. Stuttgart, Kohlhammer, 2006.
Schulte D: Diagnostik in der Verhaltenstherapie München, Urban und Schwarzenberg, 1974.

Schumacher J, Klaiberg A, Brähler E (eds): Diagnostische Verfahren zur Lebensqualität und Wohlbefinden. Göttingen, Hogrefe, 2003.

Spitzer C: Psychometrische Diagnostik dissoziativer Symptome und Störungen; in Eckhardt-Henn A, Hoffmann SO (eds): Dissoziative Bewußtseinsstörungen: Theorie, Symptomatik, Therapie. Stuttgart, Schattauer, 2004, pp 311-320.

Spitzer C, Stieglitz RD, Freyberger HJ: Fragebogen zu Dissoziativen Symptomen (FDS), ed 2. Bern, Huber, 2005.

Stangier U, Heidenreich T, Peitz M: Soziale Phobien, ed 2. Weinheim, Beltz, 2009.

Steck P: Differentieller Depressions-Fragebogen (DDF). Frankfurt, Pearson, 1998.

Steyer R, Schwenkmezger P, Notz P, Eid M: Der Mehrdimensionale Befindlichkeitsfragebogen (MDBF). Göttingen, Hogrefe, 1997

Stieglitz RD: Diagnostik und Klassifikation in der Psychiatrie. Stuttgart, Kohlhammer, 2008.

Stieglitz RD: Psychiatrische Ratingskalen in der Routinediagnostik. Psychiatr Psychother Up2date 2012; 6:177-190.

Stieglitz RD, Baumann U: Veränderungsmessung; in Stieglitz RD, Baumann U, Freyberger HJ (eds): Psychodiagnostik in Klinischer Psychologie, Psychiatrie, Psychotherapie. Stuttgart, Thieme, 2001, pp 21-38.

Stieglitz RD, Baumann U, Freyberger HJ (eds): Psychodiagnostik psychischer Störungen in Klinischer Psychologie, Psychiatrie, Psychotherapie. Stuttgart, Thieme, 2001.

Stieglitz RD, Hiller W: Strategien und Instrumente der Veränderungsmessung. Z Psychiatr Psychol Psychother 2014;im Druck.

Stieglitz RD, Smolka M, Bech P, Helmchen H: BechRafaelsen-Melancholie-Skala (BRMS). Göttingen, Hogrefe, 1998.

Strauß B, Schumacher J. (eds): Klinische Interviews und Ratingskalen. Göttingen, Hogrefe, 2005.

Strupp HH, Horowitz LJ, Lambert MJ (eds): Measuring Patient Change in Mood, Anxiety, and Personality Disorders: Toward a Core Battery. Washington D.C., American Psychological Association Press, 1997.

Vermeersch DA, Lambert MJ, Burlingame GM: Outcome Questionnaire: item sensitivity to change. J Pers Assess 2000;74:242-261.

Voderholzer U, Neumayer C, Stieglitz RD: Fremdund Selbstbeurteilungsverfahren bei der Diagnostik und Therapie psychischer Erkrankungen; in Voderholzer U, Hohagen F (eds): Therapie psychischer Erkrankungen, ed 9. München, Urban und Fischer, 2014, pp 475-491.

Waskow JE, Parloff MB: Psychotherapy Change Measures. Rockville, National Institute of Menta Health, 1975.

Watzke B, Schulz H, Dirmaier J: Zielorientierte Veränderungsmessungen als Möglichkeit einer individualisierten Ergebnisevaluation in der Psychotherapie. Z Psychiatr Psychol Psychother 2014;im Druck.

Zerssen, D v, Petermann F: Beschwerden-Liste - Revidierte Fassung (B-LR). Göttingen, Hogrefe, 2011a.

Zerssen D v, Petermann F: Befindlichkeits-Skala Revidierte Fassung (Bf-SR). Göttingen, Hogrefe, 2011b.

Zielke M, Kopf-Mehnert C: Veränderungsfragebogen des Erlebens und Verhaltens (VEV). Weinheim, Beltz, 1978. 